Research Article

\title{
Hyers-Ulam Stability of the First-Order Matrix Differential Equations
}

\author{
Soon-Mo Jung \\ Mathematics Section, College of Science and Technology, Hongik University, Sejong 30016, Republic of Korea \\ Correspondence should be addressed to Soon-Mo Jung; smjung@hongik.ac.kr \\ Received 8 September 2015; Accepted 8 November 2015 \\ Academic Editor: Richard I. Avery \\ Copyright (C) 2015 Soon-Mo Jung. This is an open access article distributed under the Creative Commons Attribution License, which \\ permits unrestricted use, distribution, and reproduction in any medium, provided the original work is properly cited. \\ We prove the generalized Hyers-Ulam stability of the first-order linear homogeneous matrix differential equations $\vec{y}^{\prime}(t)=\mathbf{A}(t) \vec{y}(t)$. \\ Moreover, we apply this result to prove the generalized Hyers-Ulam stability of the $n$th order linear differential equations with \\ variable coefficients.
}

\section{Introduction}

For a given positive integer $n$, let $I$ be a nondegenerate interval of $\mathbb{R}$. We will consider the (linear) differential equation of $n$th order

$$
\mathscr{F}\left(y^{(n)}, y^{(n-1)}, \ldots, y^{\prime}, y, x\right)=0
$$

defined on $I$, where $y: I \rightarrow \mathbb{C}$ is an $n$ times continuously differentiable function.

For an arbitrary $\varepsilon>0$, assume that an $n$ times continuously differentiable function $y: I \rightarrow \mathbb{C}$ satisfies the differential inequality

$$
\left|\mathscr{F}\left(y^{(n)}, y^{(n-1)}, \ldots, y^{\prime}, y, x\right)\right| \leq \varepsilon
$$

for all $x \in I$. If for each function $y: I \rightarrow \mathbb{C}$, satisfying inequality (2), there exists a solution $y_{0}: I \rightarrow \mathbb{C}$ of the differential equation (1) such that

$$
\left|y(x)-y_{0}(x)\right| \leq K(\varepsilon)
$$

for any $x \in I$, where $K(\varepsilon)$ depends on $\varepsilon$ only and satisfies $\lim _{\varepsilon \rightarrow 0} K(\varepsilon)=0$, then we say that the differential equation (1) satisfies (or has) the Hyers-Ulam stability (or the local HyersUlam stability if the domain $I$ is not the whole space $\mathbb{R})$. If the above statement also holds when we replace $\varepsilon$ and $K(\varepsilon)$ with some appropriate $\varphi(x)$ and $\Phi(x)$, respectively, then we say that the differential equation (1) satisfies the generalized
Hyers-Ulam stability (or the Hyers-Ulam-Rassias stability). For more detailed definition of the Hyers-Ulam stability, refer to [1].

Obłoza seems to be the first author who investigated the Hyers-Ulam stability of linear differential equations (see [2, $3])$. Given real-valued constants $a$ and $b$, let $g, r:(a, b) \rightarrow \mathbb{R}$ be continuous functions with $\int_{a}^{b}|g(x)| d x<\infty$. Assume that $\varepsilon>0$ is an arbitrary real number. Obłoza proved that if a differentiable function $y:(a, b) \rightarrow \mathbb{R}$ satisfies the inequality $\left|y^{\prime}(x)+g(x) y(x)-r(x)\right| \leq \varepsilon$ for all $x \in(a, b)$ and if a function $y_{0}:(a, b) \rightarrow \mathbb{R}$ satisfies $y_{0}^{\prime}(x)+g(x) y_{0}(x)=r(x)$ for all $x \in(a, b)$ and $y(\tau)=y_{0}(\tau)$ for some $\tau \in(a, b)$, then there exists a constant $\delta>0$ such that $\left|y(x)-y_{0}(x)\right| \leq \delta$ for all $x \in(a, b)$.

Thereafter, Alsina and Ger [4] proved that if a differentiable function $y:(a, b) \rightarrow \mathbb{R}$ satisfies the differential inequality $\left|y^{\prime}(x)-y(x)\right| \leq \varepsilon$, then there exists a function $y_{0}:(a, b) \rightarrow \mathbb{R}$ such that $y_{0}^{\prime}(x)=y_{0}(x)$ and $\mid y(x)-$ $y_{0}(x) \mid \leq 3 \varepsilon$ for all $x \in(a, b)$. This result of Alsina and Ger was generalized by Takahasi et al. [5]. Indeed, they proved the Hyers-Ulam stability of the Banach space valued differential equation $y^{\prime}(x)=\lambda y(x)$ (see also [6-12]).

In this paper, we prove the generalized Hyers-Ulam stability of the first-order linear homogeneous matrix differential equations:

$$
\vec{y}^{\prime}(t)=\mathbf{A}(t) \vec{y}(t)
$$


Moreover, we apply this result to prove the generalized HyersUlam stability of the $n$th order linear differential equations with variable coefficients.

\section{Preliminaries}

In what follows, let $I=(a, b)$ be an arbitrary open interval with $-\infty \leq a<b \leq \infty$ and let $n$ be a fixed integer larger than 1. The first-order linear inhomogeneous differential equation with matrix coefficient is of the form

$$
\vec{y}^{\prime}(t)=\mathbf{A}(t) \vec{y}(t)+\vec{f}(t)
$$

where $\vec{y}: I \rightarrow \mathbb{C}^{n}$ is an unknown vector function to be determined, $\mathbf{A}(t)$ is an $(n \times n)$ matrix whose entries are complex-valued continuous functions on $I$, and $\vec{f}: I \rightarrow \mathbb{C}^{n}$ is a continuous column vector function.

According to [13, Section 15], if the coefficient matrix $\mathbf{A}(t)$ is an $(n \times n)$ matrix whose entries are complex-valued continuous functions, then there exist $n$ linearly independent solutions $\vec{y}_{1}, \ldots, \vec{y}_{n}$ to the homogeneous matrix differential equation (4) and each of its solutions can be written in the form

$$
\vec{y}(t)=Y(t) \vec{\eta}
$$

where $\vec{\eta}=\left(\begin{array}{llll}\eta_{1} & \eta_{2} & \cdots & \eta_{n}\end{array}\right)^{\mathrm{tr}}$ is a constant column vector and $Y(t)$ is a fundamental matrix whose columns consist of $n$ linearly independent solutions $\vec{y}_{1}(t), \ldots, \vec{y}_{n}(t)$ to the homogeneous differential equation (4); that is, $Y(t)$ is an $(n \times n)$ matrix defined by

$$
Y(t)=\left(\begin{array}{llll}
\vec{y}_{1}(t) & \vec{y}_{2}(t) & \cdots & \vec{y}_{n}(t)
\end{array}\right) .
$$

In view of [13, Section 16], the general solution to the inhomogeneous differential equation (5) is given by

$$
\vec{y}(t)=Y(t) \vec{\eta}+Y(t) \int_{c}^{t} Y^{-1}(\tau) \vec{f}(\tau) d \tau,
$$

where $Y(t)$ is a fundamental matrix for the corresponding homogeneous differential equation (4) and $c$ is an arbitrarily given real number included in $I$.

Let $\left(\mathbb{C}^{n},\|\cdot\|_{n}\right)$ be a complex normed space, where each of its elements is a column vector, and let $\mathbb{C}^{n \times n}$ be a vector space consisting of all $(n \times n)$ complex matrices. We choose a norm $\|\cdot\|_{n \times n}$ on $\mathbb{C}^{n \times n}$ which is compatible with $\|\cdot\|_{n}$; that is, both norms obey

$$
\begin{aligned}
\|\mathbf{A B}\|_{n \times n} & \leq\|\mathbf{A}\|_{n \times n}\|\mathbf{B}\|_{n \times n}, \\
\|\mathbf{A} \vec{y}\|_{n} & \leq\|\mathbf{A}\|_{n \times n}\|\vec{y}\|_{n}
\end{aligned}
$$

for all $\mathbf{A}, \mathbf{B} \in \mathbb{C}^{n \times n}$ and $\vec{y} \in \mathbb{C}^{n}$.

Some of the most important matrix norms are induced by $p$-norms. For $1 \leq p \leq \infty$, the matrix norm induced by the p-norm,

$$
\|\mathbf{A}\|_{p}:=\sup _{\vec{x} \neq \overrightarrow{0}} \frac{\|\mathbf{A} \vec{x}\|_{p}}{\|\vec{x}\|_{p}}
$$

is called the matrix $p$-norm. For example, we get

$$
\begin{aligned}
\|\mathbf{A}\|_{1} & =\max _{1 \leq j \leq n} \sum_{i=1}^{n}\left|a_{i j}\right|, \\
\|\mathbf{A}\|_{\infty} & =\max _{1 \leq i \leq n} \sum_{j=1}^{n}\left|a_{i j}\right| .
\end{aligned}
$$

It is known that the matrix $p$-norm, together with the $p$ norm, satisfies the conditions in (9), where

$$
\begin{aligned}
\|\vec{x}\|_{1} & =\sum_{j=1}^{n}\left|x_{j}\right|, \\
\|\vec{x}\|_{\infty} & =\max _{1 \leq j \leq n}\left|x_{j}\right|
\end{aligned}
$$

for any $\vec{x} \in \mathbb{C}^{n}$.

\section{Generalized Hyers-Ulam Stability of (4)}

We prove the main theorem of this paper concerning the generalized Hyers-Ulam stability of the first-order linear homogeneous matrix differential equation (4).

Theorem 1. Assume that $I=(a, b)$ is an arbitrary open interval with $-\infty \leq a<b \leq \infty$. Given a fixed integer $n>1$, let $\left(\mathbb{C}^{n},\|\cdot\|_{n}\right)$ and $\left(\mathbb{C}^{n \times n},\|\cdot\|_{n \times n}\right)$ be complex normed spaces with the property (9), whose elements are column vectors, respectively, $(n \times n)$ complex matrices. Assume that the coefficient matrix $\mathbf{A}(t)$ is an $(n \times n)$ matrix whose entries are complex-valued continuous functions on $I$. Let $c$ be an arbitrary real number included in $I$ and $\varphi: I \rightarrow[0, \infty)$ a continuous function. If a continuously differentiable vector function $\vec{y}: I \rightarrow \mathbb{C}^{n}$ satisfies the inequality

$$
\left\|\vec{y}^{\prime}(t)-\mathbf{A}(t) \vec{y}(t)\right\|_{n} \leq \varphi(t)
$$

for all $t \in I$, then there exists a solution $\vec{y}_{c}: I \rightarrow \mathbb{C}^{n}$ to the homogeneous matrix differential equation (4) such that

$$
\left\|\vec{y}(t)-\vec{y}_{c}(t)\right\|_{n} \leq\|Y(t)\|_{n \times n}\left|\int_{c}^{t}\left\|Y^{-1}(\tau)\right\|_{n \times n} \varphi(\tau) d \tau\right|
$$

for all $t \in I$, where $Y(t)$ is a fundamental matrix of the homogeneous matrix differential equation (4).

Proof. If we define a continuous vector function $\vec{f}: I \rightarrow \mathbb{C}^{n}$ by $\vec{f}(t):=\vec{y}^{\prime}(t)-\mathbf{A}(t) \vec{y}(t)$, then it follows from (13) that

$$
\begin{aligned}
\|\vec{f}(t)\|_{n} & =\left\|\vec{y}^{\prime}(t)-\mathbf{A}(t) \vec{y}(t)\right\|_{n} \leq \varphi(t), \\
\vec{y}^{\prime}(t) & =\mathbf{A}(t) \vec{y}(t)+\vec{f}(t)
\end{aligned}
$$

for all $t \in I$.

In view of (5) and (8), we have

$$
\vec{y}(t)=Y(t) \vec{\eta}+Y(t) \int_{c}^{t} Y^{-1}(\tau) \vec{f}(\tau) d \tau
$$


for all $t \in I$, where $\vec{\eta}=\left(\begin{array}{lll}\eta_{1} & \cdots & \eta_{n}\end{array}\right)^{\text {tr }}$ is a constant column vector and $Y(t)$ is a fundamental matrix whose columns consist of $n$ linearly independent solutions to the homogeneous equation (4) (see [13, Section 16]).

If we define a continuously differentiable vector function $\vec{y}_{c}: I \rightarrow \mathbb{C}^{n}$ by $\vec{y}_{c}(t):=Y(t) \vec{\eta}$ for each $t \in I$, then $\vec{y}_{c}$ is a solution to the homogeneous matrix differential equation (4) in view of (6). (We know that every function $\vec{y}_{c}(t)=Y(t) \vec{\eta}$ is a solution to (4).) Moreover, by (9) and (15), we have

$$
\begin{aligned}
& \left\|\vec{y}(t)-\vec{y}_{c}(t)\right\|_{n}=\left\|Y(t) \int_{c}^{t} Y^{-1}(\tau) \vec{f}(\tau) d \tau\right\|_{n} \\
& \leq\|Y(t)\|_{n \times n}\left\|\int_{c}^{t} Y^{-1}(\tau) \vec{f}(\tau) d \tau\right\|_{n} \\
& \leq\|Y(t)\|_{n \times n}\left|\int_{c}^{t}\left\|Y^{-1}(\tau)\right\|_{n \times n}\|\vec{f}(\tau)\|_{n} d \tau\right| \\
& \leq\|Y(t)\|_{n \times n}\left|\int_{c}^{t}\left\|Y^{-1}(\tau)\right\|_{n \times n} \varphi(\tau) d \tau\right|
\end{aligned}
$$

for any $t \in I$.

If we set $n=2, \mathbf{A}(t) \equiv \mathbf{A}$ (a constant matrix), and $I=$ $(a, b)$ with $-\infty \leq a<0<b \leq \infty$, then Theorem 1 yields the following corollary.

Corollary 2. Let $\left(\mathbb{C}^{2},\|\cdot\|_{2}\right)$ and $\left(\mathbb{C}^{2 \times 2},\|\cdot\|_{2 \times 2}\right)$ be complex normed spaces with property (9), whose elements are column vectors, respectively, $(2 \times 2)$ complex matrices. Assume that the coefficient matrix $\mathbf{A}$ is given by

$$
\mathbf{A}:=\left(\begin{array}{ll}
a_{11} & a_{12} \\
a_{21} & a_{22}
\end{array}\right),
$$

where $a_{i j}$ 's are complex numbers with

$$
\begin{aligned}
& a_{11} a_{22}-a_{12} a_{21} \neq 0, \\
& \frac{\left(a_{11}+a_{22}\right)^{2}}{a_{11} a_{22}-a_{12} a_{21}} \neq 4 .
\end{aligned}
$$

Assume that $I=(a, b)$ is an arbitrary open interval with $-\infty \leq a<b \leq \infty$ and $c$ is an arbitrary real number included in $I$. Let $\varphi: I \rightarrow[0, \infty)$ be a continuous function. If a continuously differentiable vector function $\vec{y}: I \rightarrow \mathbb{C}^{2}$ satisfies the inequality

$$
\left\|\vec{y}^{\prime}(t)-\mathbf{A} \vec{y}(t)\right\|_{2} \leq \varphi(t)
$$

for all $t \in I$, then there exists a solution $\vec{y}_{c}: I \rightarrow \mathbb{C}^{2}$ to the homogeneous matrix differential equation (4) with $\mathbf{A}(t) \equiv \mathbf{A}$ such that

$$
\begin{aligned}
\left\|\vec{y}(t)-\vec{y}_{c}(t)\right\|_{2} \leq & \|\mathbf{P}\|_{2 \times 2}\left\|\mathbf{P}^{-1}\right\|_{2 \times 2}\|Z(t)\|_{2 \times 2} \\
& \cdot\left|\int_{c}^{t}\left\|Z^{-1}(\tau)\right\|_{2 \times 2} \varphi(\tau) d \tau\right|
\end{aligned}
$$

for all $t \in I$, where $Z(t)$ and $Z^{-1}(t)$ are given in (33) and (34).
Proof. The complex numbers

$$
\begin{aligned}
& \lambda_{1}=\frac{a_{11}+a_{22}+\sqrt{\left(a_{11}-a_{22}\right)^{2}+4 a_{12} a_{21}}}{2}, \\
& \lambda_{2}=\frac{a_{11}+a_{22}-\sqrt{\left(a_{11}-a_{22}\right)^{2}+4 a_{12} a_{21}}}{2}
\end{aligned}
$$

are the eigenvalues of $\mathbf{A}$ and the vectors

$$
\begin{aligned}
\vec{v}_{1} & =\left(\begin{array}{c}
2 a_{12} \\
-a_{11}+a_{22}+\sqrt{\left(a_{11}-a_{22}\right)^{2}+4 a_{12} a_{21}}
\end{array}\right) \\
& =\left(\begin{array}{c}
2 a_{12} \\
2 \lambda_{1}-2 a_{11}
\end{array}\right), \\
\vec{v}_{2} & =\left(\begin{array}{c}
2 a_{12} \\
-a_{11}+a_{22}-\sqrt{\left(a_{11}-a_{22}\right)^{2}+4 a_{12} a_{21}}
\end{array}\right) \\
& =\left(\begin{array}{c}
2 a_{12} \\
2 \lambda_{2}-2 a_{11}
\end{array}\right)
\end{aligned}
$$

are the eigenvectors of $\mathbf{A}$ corresponding to $\lambda_{1}$ and $\lambda_{2}$, respectively.

In view of [14, Theorems 1.4.5 and 7.1.4], the conditions in (20) imply that $\mathbf{A}$ is invertible and $0 \neq \lambda_{1} \neq \lambda_{2} \neq 0$. Hence, the eigenvectors $\vec{v}_{1}$ and $\vec{v}_{2}$ are linearly independent.

If we set

$$
\mathbf{P}:=\left(\begin{array}{ll}
\vec{v}_{1} & \vec{v}_{2}
\end{array}\right),
$$

then $\mathbf{P}$ is invertible and

$$
\begin{aligned}
\mathbf{P}^{-1} & =\frac{1}{4 a_{12} \sqrt{\left(a_{11}-a_{22}\right)^{2}+4 a_{12} a_{21}}} \\
& \cdot\left(\begin{array}{ll}
a_{11}-a_{22}+\sqrt{\left(a_{11}-a_{22}\right)^{2}+4 a_{12} a_{21}} & 2 a_{12} \\
-a_{11}+a_{22}+\sqrt{\left(a_{11}-a_{22}\right)^{2}+4 a_{12} a_{21}} & -2 a_{12}
\end{array}\right) \\
& =\frac{1}{2 a_{12}\left(2 \lambda_{1}-a_{11}-a_{22}\right)}\left(\begin{array}{ll}
\lambda_{1}-a_{22} & a_{12} \\
\lambda_{1}-a_{11} & -a_{12}
\end{array}\right) .
\end{aligned}
$$

Due to a well known theorem of linear algebra, it holds that

$$
\begin{aligned}
\mathbf{P}^{-1} \mathbf{A P} & =\left(\begin{array}{cc}
\lambda_{1} & 0 \\
0 & \lambda_{2}
\end{array}\right)=: \mathbf{D}, \\
\text { or } \mathbf{A} & =\mathbf{P D P}^{-1} .
\end{aligned}
$$

If we define a vector function $\vec{z}: I \rightarrow \mathbb{C}^{2}$ by

$$
\vec{z}(t):=\mathbf{P}^{-1} \vec{y}(t)
$$


for all $t \in I$, then $\vec{z}(t)$ is continuously differentiable, and it follows from (21) and (27) that

$$
\begin{aligned}
\left\|\vec{z}^{\prime}(t)-\mathbf{D} \vec{z}(t)\right\|_{2} & =\left\|\mathbf{P}^{-1} \vec{y}^{\prime}(t)-\left(\mathbf{P}^{-1} \mathbf{P}\right) \mathbf{D} \mathbf{P}^{-1} \vec{y}(t)\right\|_{2} \\
& =\left\|\mathbf{P}^{-1} \vec{y}^{\prime}(t)-\mathbf{P}^{-1} \mathbf{A} \vec{y}(t)\right\|_{2} \\
& \leq\left\|\mathbf{P}^{-1}\right\|_{2 \times 2}\left\|\vec{y}^{\prime}(t)-\mathbf{A} \vec{y}(t)\right\|_{2} \\
& \leq\left\|\mathbf{P}^{-1}\right\|_{2 \times 2} \varphi(t)
\end{aligned}
$$

for all $t \in I$.

According to Theorem 1 , there exists a vector function $\vec{z}_{c}$ : $I \rightarrow \mathbb{C}^{2}$ satisfying

$$
\begin{aligned}
& \vec{z}_{c}^{\prime}(t)=\mathbf{D} \vec{z}_{c}(t) \\
& \left\|\vec{z}(t)-\vec{z}_{c}(t)\right\|_{2} \\
& \quad \leq\|Z(t)\|_{2 \times 2}\left|\int_{c}^{t}\left\|Z^{-1}(\tau)\right\|_{2 \times 2}\left\|\mathbf{P}^{-1}\right\|_{2 \times 2} \varphi(\tau) d \tau\right|
\end{aligned}
$$

for every $t \in I$, where $Z(t)$ is a fundamental matrix of the homogeneous matrix differential equation (30).

If we set

$$
\begin{aligned}
& \vec{z}_{1}(t):=\left(\begin{array}{c}
e^{\lambda_{1} t} \\
0
\end{array}\right), \\
& \vec{z}_{2}(t):=\left(\begin{array}{c}
0 \\
e^{\lambda_{2} t}
\end{array}\right),
\end{aligned}
$$

then $\vec{z}_{1}$ and $\vec{z}_{2}$ are the solutions to (30) which are linearly independent. Therefore, the fundamental matrix $Z(t)$ of the matrix differential equation (30) may be expressed as

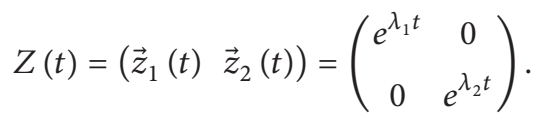

Then, we have

$$
Z^{-1}(t)=\left(\begin{array}{cc}
e^{-\lambda_{1} t} & 0 \\
0 & e^{-\lambda_{2} t}
\end{array}\right)
$$

for any $t \in I$.

If we set $\vec{y}_{c}(t):=\mathbf{P} \vec{z}_{c}(t)$ for all $t \in I$, then it follows from (27) and (30) that

$$
\begin{aligned}
\vec{y}_{c}^{\prime}(t) & =\mathbf{P} \vec{z}_{c}^{\prime}(t)=\mathbf{P D} \vec{z}_{c}(t)=\left(\mathbf{P D P} \mathbf{P}^{-1}\right) \mathbf{P} \vec{z}_{c}(t) \\
& =\mathbf{A} \vec{y}_{c}(t)
\end{aligned}
$$

for all $t \in I$; that is, $\vec{y}_{c}(t)$ is a solution to the matrix differential equation (4) with $\mathbf{A}(t) \equiv \mathbf{A}$.

Finally, it follows from (28) and (31) that

$$
\begin{aligned}
& \left\|\vec{y}(t)-\vec{y}_{c}(t)\right\|_{2}=\left\|\mathbf{P} \vec{z}(t)-\mathbf{P} \vec{z}_{c}(t)\right\|_{2} \leq\|\mathbf{P}\|_{2 \times 2} \\
& \cdot\left\|\vec{z}(t)-\vec{z}_{c}(t)\right\|_{2} \leq\|\mathbf{P}\|_{2 \times 2}\left\|\mathbf{P}^{-1}\right\|_{2 \times 2}\|Z(t)\|_{2 \times 2} \\
& \quad \cdot\left|\int_{c}^{t}\left\|Z^{-1}(\tau)\right\|_{2 \times 2} \varphi(\tau) d \tau\right|
\end{aligned}
$$

for all $t \in I$.

\section{Applications}

We now consider the linear $n$th order differential equations with variable coefficients

$$
\begin{aligned}
y^{(n)}(t) & +a_{n-1}(t) y^{(n-1)}(t)+\cdots+a_{1}(t) y^{\prime}(t) \\
& +a_{0}(t) y(t)=0
\end{aligned}
$$

where $a_{0}, a_{1}, \ldots, a_{n-1}: I \rightarrow \mathbb{C}$ are continuous functions.

In this section, we set

$$
\mathbf{A}(t):=\left(\begin{array}{ccccccc}
0 & 1 & 0 & 0 & \cdots & 0 & 0 \\
0 & 0 & 1 & 0 & \cdots & 0 & 0 \\
\vdots & \vdots & \vdots & \vdots & \ddots & \vdots & \vdots \\
0 & 0 & 0 & 0 & \cdots & 0 & 1 \\
-a_{0}(t) & -a_{1}(t) & -a_{2}(t) & -a_{3}(t) & \cdots & -a_{n-2}(t) & -a_{n-1}(t)
\end{array}\right)
$$

for all $t \in I$.

Theorem 3. Assume that $I=(a, b)$ is an arbitrary open interval with $-\infty \leq a<b \leq \infty$. For a fixed integer $n>1$, assume that continuous functions $a_{0}, a_{1}, \ldots, a_{n-1}: I \rightarrow \mathbb{C}$ are given. Let $c$ be an arbitrary real number included in $I$ and $\varphi: I \rightarrow[0, \infty)$ a continuous function. If an $n$ times continuously differentiable function $y: I \rightarrow \mathbb{C}$ satisfies the inequality

$$
\begin{aligned}
& \mid y^{(n)}(t)+a_{n-1}(t) y^{(n-1)}(t)+a_{n-2}(t) y^{(n-2)}(t)+\cdots \\
& \quad+a_{1}(t) y^{\prime}(t)+a_{0}(t) y(t) \mid \leq \varphi(t)
\end{aligned}
$$


for all $t \in I$, then there exists a solution $y_{c}: I \rightarrow \mathbb{C}$ to the homogeneous matrix differential equation (37) such that

$$
\left|y(t)-y_{c}(t)\right| \leq\|U(t)\|_{\infty}\left|\int_{c}^{t}\left\|U^{-1}(\tau)\right\|_{\infty} \varphi(\tau) d \tau\right|
$$

for all $t \in I$, where $U(t)$ is a fundamental matrix of the firstorder linear homogeneous matrix differential equation (46).

Proof. If we define continuous functions $u_{1}, \ldots, u_{n}: I \rightarrow \mathbb{C}$ by

$$
\begin{gathered}
u_{1}(t)=y(t), \\
u_{2}(t)=y^{\prime}(t), \\
\vdots \\
u_{n}(t)=y^{(n-1)}(t),
\end{gathered}
$$

then it follows from (39) and (41) that

$$
\begin{gathered}
\left|u_{1}^{\prime}(t)-u_{2}(t)\right|=0, \\
\left|u_{2}^{\prime}(t)-u_{3}(t)\right|=0, \\
\vdots \\
\left|u_{n-1}^{\prime}(t)-u_{n}(t)\right|=0
\end{gathered}
$$

and moreover

$$
\begin{aligned}
& \mid u_{n}^{\prime}(t)+a_{n-1}(t) u_{n}(t)+a_{n-2}(t) u_{n-1}(t)+\cdots \\
& \quad+a_{1}(t) u_{2}(t)+a_{0}(t) u_{1}(t)|=| y^{(n)}(t) \\
& \quad+a_{n-1}(t) y^{(n-1)}(t)+a_{n-2}(t) y^{(n-2)}(t)+\cdots \\
& \quad+a_{1}(t) y^{\prime}(t)+a_{0}(t) y(t) \mid \leq \varphi(t)
\end{aligned}
$$

for all $t \in I$. That is, we have

$$
\left\|\vec{u}^{\prime}(t)-\mathbf{A}(t) \vec{u}(t)\right\|_{\infty} \leq \varphi(t)
$$

for all $t \in I$, where the coefficient matrix $\mathbf{A}(t)$ is defined in (38), the vector norm $\|\cdot\|_{\infty}$ is defined in (12), and the continuously differentiable vector function $\vec{u}: I \rightarrow \mathbb{C}^{n}$ is defined by

$$
\vec{u}(t):=\left(\begin{array}{c}
u_{1}(t) \\
u_{2}(t) \\
\vdots \\
u_{n}(t)
\end{array}\right)=\left(\begin{array}{c}
y(t) \\
y^{\prime}(t) \\
\vdots \\
y^{(n-1)}(t)
\end{array}\right) .
$$

According to Theorem 1 , there exists a continuously differentiable vector function $\vec{u}_{c}: I \rightarrow \mathbb{C}^{n}$ satisfying

$$
\begin{aligned}
\vec{u}_{c}^{\prime}(t) & =\mathbf{A}(t) \vec{u}_{c}(t) \\
\left\|\vec{u}(t)-\vec{u}_{c}(t)\right\|_{\infty} \leq\|U(t)\|_{\infty}\left|\int_{c}^{t}\left\|U^{-1}(\tau)\right\|_{\infty} \varphi(\tau) d \tau\right| &
\end{aligned}
$$

for all $t \in I$, where $U(t)$ is a fundamental matrix of the firstorder linear homogeneous matrix differential equation (46).

We now set

$$
\vec{u}_{c}(t):=\left(\begin{array}{c}
u_{c, 1}(t) \\
u_{c, 2}(t) \\
\vdots \\
u_{c, n}(t)
\end{array}\right)
$$

and then we consider the first $n-1$ elements of (46) to show that

$$
\begin{gathered}
u_{c, 1}^{\prime}(t)=u_{c, 2}(t), \\
u_{c, 2}^{\prime}(t)=u_{c, 3}(t)=u_{c, 1}^{\prime \prime}(t), \\
\vdots \\
u_{c, n-1}^{\prime}(t)=u_{c, n}(t)=u_{c, 1}^{(n-1)}(t) .
\end{gathered}
$$

Moreover, we use the last equality of (49) and then the last element of (46) to see

$$
\begin{aligned}
u_{c, n}^{\prime}(t)= & u_{c, 1}^{(n)}(t) \\
= & -a_{0}(t) u_{c, 1}(t)-a_{1}(t) u_{c, 2}(t)-\cdots \\
& -a_{n-2}(t) u_{c, n-1}(t)-a_{n-1}(t) u_{c, n}(t)
\end{aligned}
$$

for all $t \in I$. The continuous differentiability of $u_{c, n}$ implies that $u_{c, 1}$ is $n$ times continuously differentiable. Using (49) and (50), we further have

$$
\begin{gathered}
u_{c, 1}^{(n)}(t)+a_{n-1}(t) u_{c, 1}^{(n-1)}(t)+\cdots+a_{1}(t) u_{c, 1}^{\prime}(t) \\
+a_{0}(t) u_{c, 1}(t)=0
\end{gathered}
$$

for every $t \in I$.

If we define a function $y_{c}: I \rightarrow \mathbb{C}$ by

$$
y_{c}(t):=u_{c, 1}(t)
$$

then it follows from (51) that the $n$ times continuously differentiable function $y_{c}$ is a solution to the homogeneous matrix differential equation (37). Moreover, by (45), (47), and (48), we get

$$
\begin{aligned}
\left|y(t)-y_{c}(t)\right| & =\left|u_{1}(t)-u_{c, 1}(t)\right| \leq\left\|\vec{u}(t)-\vec{u}_{c}(t)\right\|_{\infty} \\
& \leq\|U(t)\|_{\infty}\left|\int_{c}^{t}\left\|U^{-1}(\tau)\right\|_{\infty} \varphi(\tau) d \tau\right|
\end{aligned}
$$

for all $t \in I$.

Remark 4. Popa and Raşa [11] proved the Hyers-Ulam stability of the linear $n$th order differential equations with variable coefficients (37). For any continuous function $\lambda: I \rightarrow \mathbb{C}$, they introduced the differential operators $D_{\lambda}$ and $D^{n}$ by

$$
\begin{aligned}
D_{\lambda}(y)(t):= & y^{\prime}(t)+\lambda y(t) \\
D^{n}(y)(t):= & y^{(n)}(t)+a_{n-1}(t) y^{(n-1)}(t)+\cdots \\
& +a_{1}(t) y^{\prime}(t)+a_{0}(t) y(t)
\end{aligned}
$$


for any $n$ times continuously differentiable function $y$ : $I \rightarrow \mathbb{C}$. Under the assumptions that there exist continuous functions $r_{1}, r_{2}, \ldots, r_{n}: I \rightarrow \mathbb{C}$ such that

$$
D^{n}=D_{r_{1}} \circ D_{r_{2}} \circ \cdots \circ D_{r_{n}}
$$

and that $\inf _{t \in I}\left|r_{k}(t)\right|>0$ for every $k \in\{1,2, \ldots, n\}$, they proved the Hyers-Ulam stability of the linear $n$th order differential equations (37) (see [11, Theorem 3.1]).

We proved the generalized Hyers-Ulam stability of the linear $n$th order differential equations (37) in Theorem 3 without additional assumptions. Based on this argument, Theorem 3 gains an advantage over [11, Theorem 3.1].

However, we remark that the hypotheses in [11, Theorem 3.1] are stronger, but the conclusions are also stronger: the Hyers-Ulam constant is described in a more explicit fashion.

Finally, we introduce an example to illustrate the usefulness of Theorem 3 for proving the generalized Hyers-Ulam stability of the second-order linear differential equation

$$
y^{\prime \prime}(t)+\omega^{2} y(t)=0
$$

for a simple harmonic oscillator.

Example 5. Assume that $I=(a, b)$ is an arbitrary open interval with $-\infty \leq a<b \leq \infty$. Let $\omega$ be a positive real number, let $c$ be an arbitrary real number included in $I$, and let $\varphi: I \rightarrow[0, \infty)$ be a continuous function such that

$$
\int_{c}^{t} \varphi(\tau) d \tau
$$

exists for each $t \in I$. If a twice continuously differentiable function $y: I \rightarrow \mathbb{C}$ satisfies the inequality

$$
\left|y^{\prime \prime}(t)+\omega^{2} y(t)\right| \leq \varphi(t)
$$

for all $t \in I$, then there exists a solution $y_{c}: I \rightarrow \mathbb{C}$ to the second-order linear differential equation (56) such that

$$
\left|y(t)-y_{c}(t)\right| \leq \max \{1, \omega\} \frac{\omega+1}{\omega}\left|\int_{c}^{t} \varphi(\tau) d \tau\right|
$$

for all $t \in I$.

Proof. If we set

$$
\begin{aligned}
\mathbf{A} & :=\left(\begin{array}{cc}
0 & 1 \\
-\omega^{2} & 0
\end{array}\right), \\
\vec{u}(t) & :=\left(\begin{array}{c}
y(t) \\
y^{\prime}(t)
\end{array}\right),
\end{aligned}
$$

then it follows from (58) that

$$
\left\|\vec{u}^{\prime}(t)-\mathbf{A} \vec{u}(t)\right\|_{\infty} \leq \varphi(t)
$$

for all $t \in I$.
In view of Theorem 3, there exists a twice continuously differentiable solution $y_{c}: I \rightarrow \mathbb{C}$ to (56) such that

$$
\left|y(t)-y_{c}(t)\right| \leq\|U(t)\|_{\infty}\left|\int_{c}^{t}\left\|U^{-1}(\tau)\right\|_{\infty} \varphi(\tau) d \tau\right|
$$

for all $t \in I$, where $U(t)$ is a fundamental matrix of the matrix differential equation $\vec{u}^{\prime}(t)=\mathbf{A} \vec{u}(t)$.

We know that the eigenvalues of the matrix $\mathbf{A}$ are $\lambda_{1}=$ $-i \omega$ and $\lambda_{2}=i \omega$ and the eigenvectors of $\mathbf{A}$ corresponding to $\lambda_{1}$ and $\lambda_{2}$ are

$$
\begin{gathered}
\left(\begin{array}{c}
1 \\
-i \omega
\end{array}\right), \\
\left(\begin{array}{c}
1 \\
i \omega
\end{array}\right),
\end{gathered}
$$

respectively. If we set

$$
\begin{aligned}
& \mathbf{P}:=\left(\begin{array}{cc}
1 & 1 \\
-i \omega & i \omega
\end{array}\right), \\
& \mathbf{D}:=\left(\begin{array}{cc}
-i \omega & 0 \\
0 & i \omega
\end{array}\right),
\end{aligned}
$$

then $\mathbf{A}=\mathbf{P D P} \mathbf{P}^{-1}$ or $\mathbf{D}=\mathbf{P}^{-1} \mathbf{A P}$.

If we set $\vec{z}(t):=\mathbf{P}^{-1} \vec{u}(t)$, then it follows from (61) that

$$
\begin{aligned}
& \left\|\vec{z}^{\prime}(t)-\mathbf{D} \vec{z}(t)\right\|_{\infty} \\
& \quad=\left\|\mathbf{P}^{-1} \vec{u}^{\prime}(t)-\left(\mathbf{P}^{-1} \mathbf{A P}\right) \mathbf{P}^{-1} \vec{u}(t)\right\|_{\infty} \\
& \quad \leq\left\|\mathbf{P}^{-1}\right\|_{\infty} \varphi(t)
\end{aligned}
$$

for all $t \in I$. It is not difficult to find a fundamental matrix $Z(t)$ of the homogeneous matrix differential equation $\vec{z}^{\prime}(t)=$ $\mathbf{D} \vec{z}(t)$ :

$$
Z(t)=\left(\begin{array}{cc}
e^{-i \omega t} & 0 \\
0 & e^{i \omega t}
\end{array}\right)
$$

Using this fundamental matrix, we further get

$$
\begin{aligned}
U(t) & =\mathbf{P} Z(t)=\left(\begin{array}{cc}
e^{-i \omega t} & e^{i \omega t} \\
-i \omega e^{-i \omega t} & i \omega e^{i \omega t}
\end{array}\right), \\
U^{-1}(t) & =\frac{1}{2 i \omega}\left(\begin{array}{cc}
i \omega e^{i \omega t} & -e^{i \omega t} \\
i \omega e^{-i \omega t} & e^{-i \omega t}
\end{array}\right) .
\end{aligned}
$$

Since

$$
\begin{aligned}
\|U(t)\|_{\infty} & =\max \{2,2 \omega\}, \\
\left\|U^{-1}(t)\right\|_{\infty} & =\frac{\omega+1}{2 \omega},
\end{aligned}
$$

it follows from (62) that

$$
\left|y(t)-y_{c}(t)\right| \leq \max \{1, \omega\} \frac{\omega+1}{\omega}\left|\int_{c}^{t} \varphi(\tau) d \tau\right|
$$

for all $t \in I$. 


\section{Conflict of Interests}

The author declares that he has no competing interests.

\section{Authors' Contribution}

The author read and approved the final paper.

\section{Acknowledgments}

This research was supported by Basic Science Research Program through the National Research Foundation of Korea (NRF) funded by the Ministry of Education (no. 2013R1A1A2005557). This work was supported by 2015 Hongik University Research Fund.

\section{References}

[1] D. H. Hyers, G. Isac, and T. M. Rassias, Stability of Functional Equations in Several Variables, Birkhäuser, Boston, Mass, USA, 1998.

[2] M. Obłoza, "Hyers stability of the linear differential equation," Rocznik Naukowo-Dydaktyczny. Prace Matematyczne, vol. 13, no. 13, pp. 259-270, 1993.

[3] M. Obłoza , "Connections between Hyers and Lyapunov stability of the ordinary differential equations," Rocznik NaukowoDydaktyczny. Prace Matematyczne, vol. 14, pp. 141-146, 1997.

[4] C. Alsina and R. Ger, "On some inequalities and stability results related to the exponential function," Journal of Inequalities and Applications, vol. 2, no. 4, pp. 373-380, 1998.

[5] S.-E. Takahasi, T. Miura, and S. Miyajima, "On the Hyers-Ulam stability of the Banach space-valued differential equation $y^{\prime}=$ $\lambda y$," Bulletin of the Korean Mathematical Society, vol. 39, no. 2, pp. 309-315, 2002.

[6] G. Choi and S.-M. Jung, "Invariance of Hyers-Ulam stability of linear differential equations and its applications," Advances in Difference Equations, vol. 2015, article 277, 14 pages, 2015.

[7] S.-M. Jung, "Hyers-ulam stability of linear differential equations of first order," Applied Mathematics Letters, vol. 17, no. 10, pp. 1135-1140, 2004.

[8] S.-M. Jung, "Hyers-Ulam stability of linear differential equations of first order, II," Applied Mathematics Letters, vol. 19, no. 9, pp. 854-858, 2006.

[9] T. Miura, H. Oka, S.-E. Takahasi, and N. Niwa, "Hyers-Ulam stability of the first order linear differential equation for Banach space-valued holomorphic mappings," Journal of Mathematical Inequalities, vol. 1, no. 3, pp. 377-385, 2007.

[10] D. Popa and I. Raşa, "On the Hyers-Ulam stability of the linear differential equation," Journal of Mathematical Analysis and Applications, vol. 381, no. 2, pp. 530-537, 2011.

[11] D. Popa and I. Raşa, "Hyers-Ulam stability of the linear differential operator with nonconstant coefficients," Applied Mathematics and Computation, vol. 219, no. 4, pp. 1562-1568, 2012.

[12] G. Wang, M. Zhou, and L. Sun, "Hyers-Ulam stability of linear differential equations of first order," Applied Mathematics Letters, vol. 21, no. 10, pp. 1024-1028, 2008.

[13] W. Walter, Ordinary Differential Equations, Springer, New York, NY, USA, 1998.

[14] H. Anton, Elementary Linear Algebra, John Wiley \& Sons, 9th edition, 2005. 


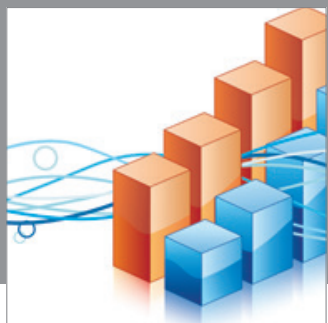

Advances in

Operations Research

mansans

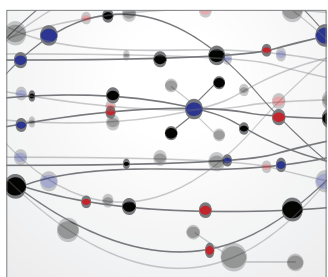

The Scientific World Journal
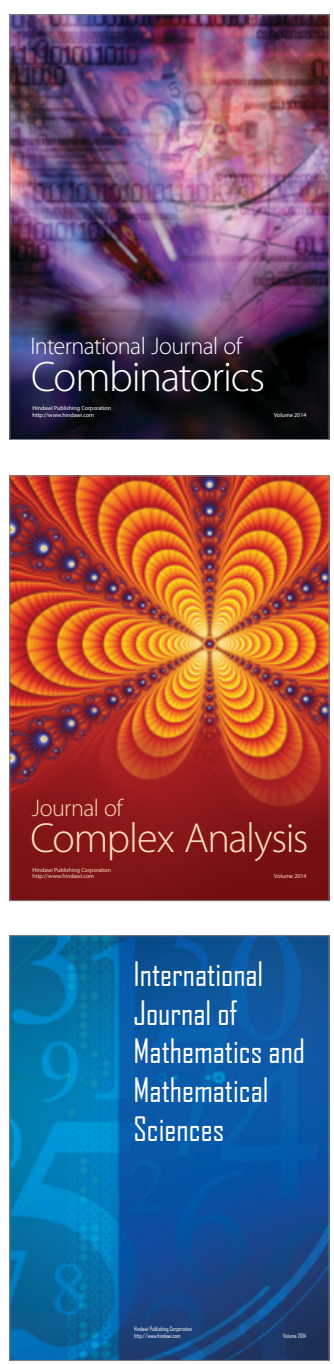
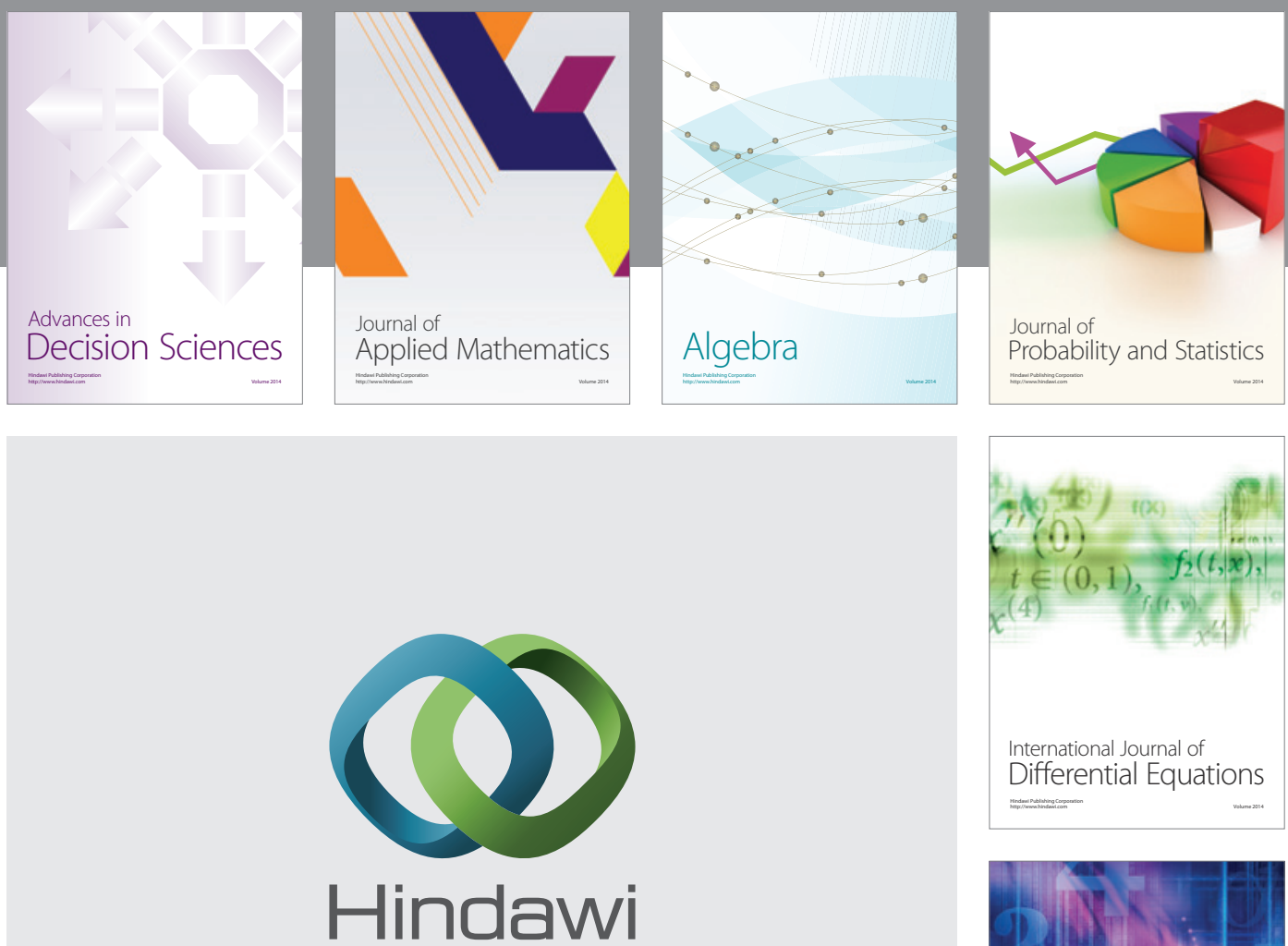

Submit your manuscripts at http://www.hindawi.com
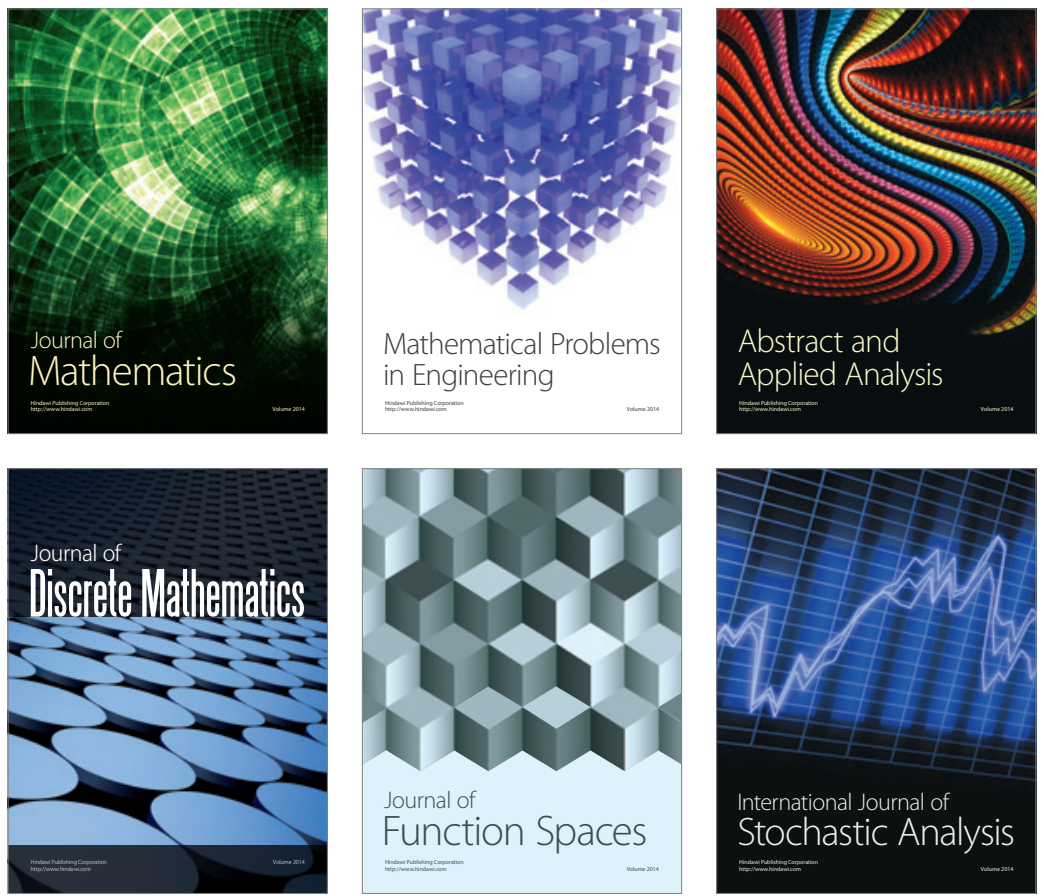

Journal of

Function Spaces

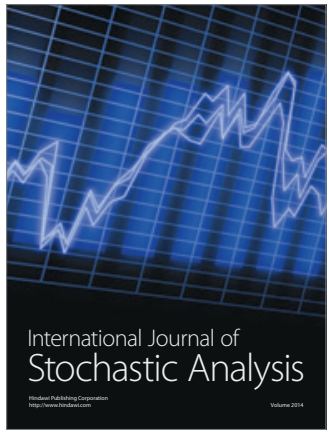

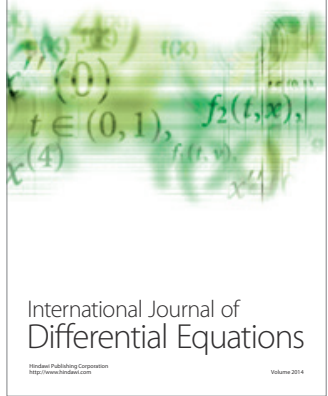
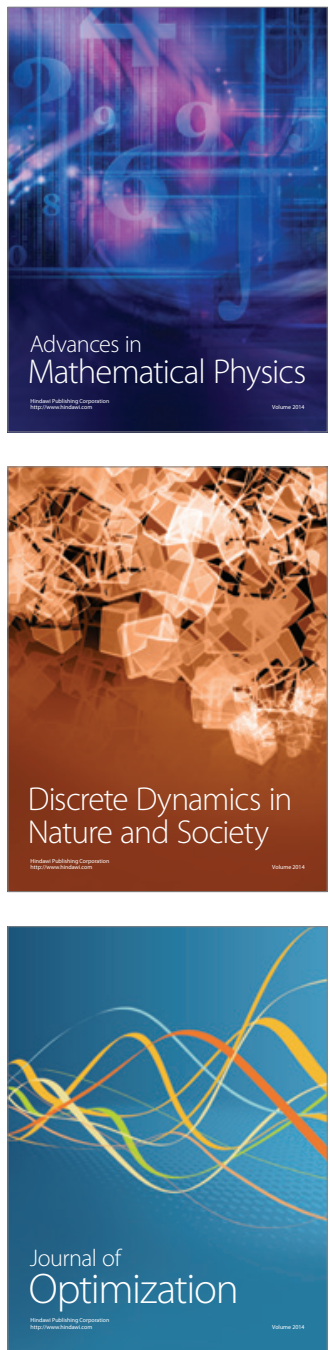